\title{
Expectancies generated by melodic intervals: Perceptual judgments of melodic continuity
}

\author{
LOLA L. CUDDY and CAROLE A. LUNNEY \\ Queen's University, Kingston, Ontario, Canada
}

\begin{abstract}
The present study tested quantified predictors based on the bottom-up principles of Narmour's (1990) implication-realization model of melodic expectancy against continuity ratings collected for a tone that followed a two-tone melodic beginning. Twenty-four subjects ( 12 musically trained, 12 untrained) were presented with each of eight melodic intervals-two successive tones which they were asked to consider as the beginning of a melody. On each trial, a melodic interval was followed by a third tone, one of the 25 chromatic notes within the range one octave below to one octave above the second tone of the interval. The subjects were asked to rate how well the third tone continued the melody. A series of regression analyses was performed on the continuation ratings, and a final model to account for the variance in the ratings is proposed. Support was found for three of Narmour's principles and a modified version of a fourth. Support was also found for predictor variables based on the pitch organization of tonal harmonic music. No significant differences between the levels of musical training were encountered.
\end{abstract}

The role of expectation, or the anticipation of future events, has stimulated psychological theory and research since the late 19th century (Carlsen, 1990). Recent research related to expectancy has been conducted in areas as varied as linguistic processing (Aubel \& Franks, 1983; Mills, 1980), story comprehension and memory (Bransford \& Johnson, 1972), and visual perception (Coren, Porac, \& Theodor, 1986). With respect to music, the concept of expectancy has been investigated in a number of different contexts, including dynamic attending to music (Jones, 1981, 1982, 1990), time estimation (Jones \& Boltz, 1989; Dowling, 1990), the generation of temporal and melodic expectancies (Boltz, 1993), and the influence of melodic and harmonic expectancies on perception and performance (Schmuckler, 1989, 1990). The present study continues the tradition of this research, with specific focus on Narmour's (1990) implicationrealization model of musical expectancy.

Narmour (1990) proposes a music-theoretic approach to melodic expectancy in which the influences of two perceptual systems - top-down and bottom-up processesare elaborated. We acknowledge that there are critical issues involved in the categorization of perceptual processes in this way (see, e.g., Dodwell, 1993) but we retain Narmour's terminology for two reasons. First, Narmour

The data were presented in an honor's thesis submitted by C.A.L. to the Department of Psychology, Queen's University, Kingston. Research was supported by an operating grant to L.L.C. and an Undergraduate Summer Award to C.A.L. from the Natural Sciences and Engineering Council of Canada. We thank C. L. Krumhansl for her invaluable advice and support of this research, J. C. Carlsen for permission to access the data from Carlsen (1981), and the reviewers of an earlier version of this manuscript. Requests for reprints should be sent to L. L. Cuddy, Department of Psychology, Queen's University, Kingston, ON, Canada K7L 3N6. identifies the bottom-up processes with quantitative principles that are amenable to psychological test. In the present study, we evaluate the quantification and application of five bottom-up principles. Second, Narmour's distinction between processes is compatible with two different focuses of research in the psychology of music and, indeed, in psychology in general - focus on "the use of sensory information in pattern recognition" as distinct from focus on "the use of the context of the pattern as well as general knowledge in recognition" (Anderson, 1990, p. 48). Narmour's distinction between bottom-up and top-down processes is also compatible with Dowling's (1990) description of two kinds of expectancies: those which "our perceptual systems and brain expect and process automatically" and those which are "the natural outgrowth of our abstracted, explicit knowledge of the world" (p. 148).

Research on bottom-up processes of perception has been strongly influenced by the work of Gestalt psychologists, who proposed that we respond innately and automatically to organized patterns of sensory input without influence from learning (Wertheimer, 1923, cited in Deutsch, 1982). Traditional Gestaltists explain perceptual grouping by presuming the recognition of preexisting, unified wholes, whose structure and meaning are defined by the operation of the nervous system rather than by the sensations themselves. The Gestalt law of Prägnanz (good figure) states that psychological organization will always be as "good" as possible. The main problem associated with this "holistic" approach is the difficulty of providing a clear, unambiguous, noncircular, and hence testable definition of "goodness" of form (Pomerantz, 1981).

However, according to Meyer (1956), "it is possible to accept the empirical data, the laws, discovered by Gestalt 
psychologists without adopting the hypothetical explanations furnished by the theory" (p. 83). The laws to which Meyer is referring consist of a number of grouping principles that operate at a local level. These include proximity, defined as the tendency for elements near to each other to be grouped together; similarity, the tendency for elements that share important physical attributes to be grouped together; and common fate, the tendency for elements moving in the same direction to be grouped together (Schiffman, 1990). These bottom-up principles have the advantage of being objectively definable and empirically testable (Garner, 1981; Pomerantz, 1981). Although originally conceptualized in terms of visual perception, the concepts have been successfully adapted to explain auditory grouping phenomena, such as the emergence of melodic lines, grouping by timbre, and a number of auditory illusions (Bregman, 1990; Deutsch, 1982; Dowling \& Harwood, 1986).

In addition to these perceptual grouping principles, the perception of music is also influenced by learned or top-down processes. It has been proposed that, through specific musical training as well as informal experience, listeners acquire knowledge about the structural significance and relative importance of elements within the music of their culture (Krumhansl, 1990). The influence of learning on music perception can be addressed by comparing aspects of listeners' responses to music with the formal organizational structure described by music theory (see, e.g., Berry, 1976).

Much of the research in this area has focused on the Western tonal-harmonic idiom because of the wide availability of listeners with extensive exposure to this music and the large amount of music-theoretic work associated with it (Krumhansl, 1990). Of particular relevance to the study of melody is the traditional description of the organization of notes with respect to a given key (Meyer, 1956). The principal feature of this organization is the categorization of notes into four levels of structural importance and stability. First, the tonic (doh) is considered the most important and stable note. It is followed, second, by the other notes of the tonic triad (the fifth note of the scale, called the dominant, and the third note of the scale, called the mediant). Third in importance are the remaining scale notes in the key of the tonic. Fourth, least important and least stable, are the notes outside the key. An obvious question is whether listeners are sensitive to and are influenced by this "tonal hierarchy" (Krumhansl, 1990). Studies using a probetone technique, in which subjects are asked to rate the goodness-of-fit of each note of the chromatic scale relative to a key-defining context, have provided evidence to support the existence of a cognitive schema corresponding to the hierarchy defined by music theory (e.g., Cuddy \& Badertscher, 1987; Krumhans1, 1990; Krumhansi \& Kessler, 1982). This abstract system of knowledge gained through exposure to a musical style may facilitate memory, comprehension, and performance of music and may lead to expectations about future events (Palmer \& Krumhansl, 1990).

\section{Narmour's Theory of Musical Expectancy}

Eugene Narmour's (1990) theoretical work has its roots in Leonard Meyer's (1956) theory of affective responses to music. Meyer proposes that the same basic psychological processes underlie all affective responses, including those to music. He explains that emotion is aroused "when an expectation-a tendency to respondactivated by the music stimulus situation, is temporarily or permanently blocked" (p. 31). In addition to denying expectancies, music provides meaningful and satisfying resolutions for these denials; resolutions are pleasing. Expectations are developed "in connection with particular musical styles and of the modes of human perception, cognition, and response - the psychological laws of mental life" (p. 30).

Building on Meyer's work, Narmour (1989, 1990, 1991) developed the implication-realization model; as noted above, it outlines both bottom-up processes of expectation, which are "automatic, unconscious [and] preprogrammed" (Narmour, 1991, p. 3), and top-down processes, which are influenced by prior learning. The bottom-up system generates expectancies based on Gestalt-like principles. Although Narmour draws heavily on Gestalt theory, he attempts to avoid logical difficulties by rejecting the Gestalt premise of preexisting wholes, while maintaining the testable concepts of local pattern organization. The bottom-up principles are assumed to be universal in their generation of melodic expectancies, although their presence may be modified by stylistic factors. Narmour (1990) argues that the topdown and bottom-up systems are separate and independent so that conflicting expectations may be generated both between and within them.

Narmour's implication-realization model deals with many complex aspects of musical structure and perception. The present article deals with a specific component of the model - the description and quantification of lowlevel, tone-to-tone expectancies for melodies. Two successive tones of a melody form what is called a melodic interval, and, according to the model, melodic intervals vary in the degree of nonclosure which they convey. An unclosed interval, termed an implicative interval, is said to generate expectancies for what tone will continue the melody. The tone that follows the unclosed interval forms a melodic interval between the second and third tone, termed the realized interval. The expectancies generated by the implicative interval have been operationalized by Narmour (1990) as five bottom-up, Gestaltbased principles of continuation: registral direction, intervallic difference, registral return, proximity, and closure.

Many of Narmour's basic concepts have been outlined in an elegant summary by Krumhansl (1995; see also Krumhansl, 1991), from which we have selected those which apply directly to the present study. Table 1 provides a general definition of each of the five bottom-up principles; the definitions have been abstracted from Krumhans1 (1995). Figure 1 (from Krumhansl, 1995) shows a corresponding diagram for each of the five prin- 
ciples along with the quantitative predictor variables used in the data analysis of the present research. Each diagram in Figure 1 is a grid with the size of the implicative interval represented along the vertical dimension and the size and direction of the realized interval represented along the horizontal dimension. The numerical code within the diagram represents, according to the model, the degree to which the realized interval fulfills the expectancy generated by the implicative interval.

For every size of implicative interval, each possible realized interval can be classified as to whether or not it fulfills the implications generated by the five different bottom-up principles. The implicative intervals are divided into "small" intervals, of five semitones or less, and "large" intervals, of seven semitones or greater. (The implicative interval of six semitones-the tritone-must be disambiguated by context, and is not studied here.)

As an example, consider the implicative interval $\mathrm{C}_{4}-$ $\mathrm{Eb}_{4}$. This is an interval of three semitones, called a minor third, and it is considered a "small" interval. Suppose it is followed by the note $F_{4}$, which forms a realized interval $\left(E b_{4}-F_{4}\right)$ of two semitones, called a major second. The realized interval continues the pitch direction (ascending) of the implicative interval. Each interval may be located on the appropriate axis of each chart of Figure 1. Next, the intersection of the two intervals may be

Table 1

Narmour's (1990) Bottom-Up Principles of Melodic Expectancy

\begin{tabular}{lll}
\hline Principle & Implication Generated & \multicolumn{1}{c}{ Definition } \\
\hline $\begin{array}{l}\text { Registral } \\
\text { direction }\end{array}$ & $\begin{array}{l}\text { Small intervals imply a } \\
\text { continuation in the same } \\
\text { direction }\end{array}$ & $\begin{array}{l}\text { Up-up, down-down, and } \\
\text { lateral-lateral patterns of } \\
\text { implicative and realized } \\
\text { Large intervals imply a } \\
\text { contervals are considered to } \\
\text { site direction }\end{array}$
\end{tabular}

Intervallic Small intervals imply a difference realized interval of a similar size

Large intervals imply a realized interval of a smaller size

Registral Implies a return to the return pitch region of first note of the implicative interval for both small and large intervals

Proximity A general implication for small intervals

Closure Closure is realized (1) when pitch contour changes direction, and/or (2) when a large interval is followed by a smaller one
A similarly sized continuation is \pm 3 semitones when direction is the same, \pm 2 semitones when direction changes

A continuation of within two semitones of the first note of the implicative interval is considered a return

A continuation of five semitones or less is considered proximate

There are three levels corresponding to fulfillment of neither Condition 1 nor Condition 2, either Condition 1 or Condition 2, or both Conditions 1 and 2
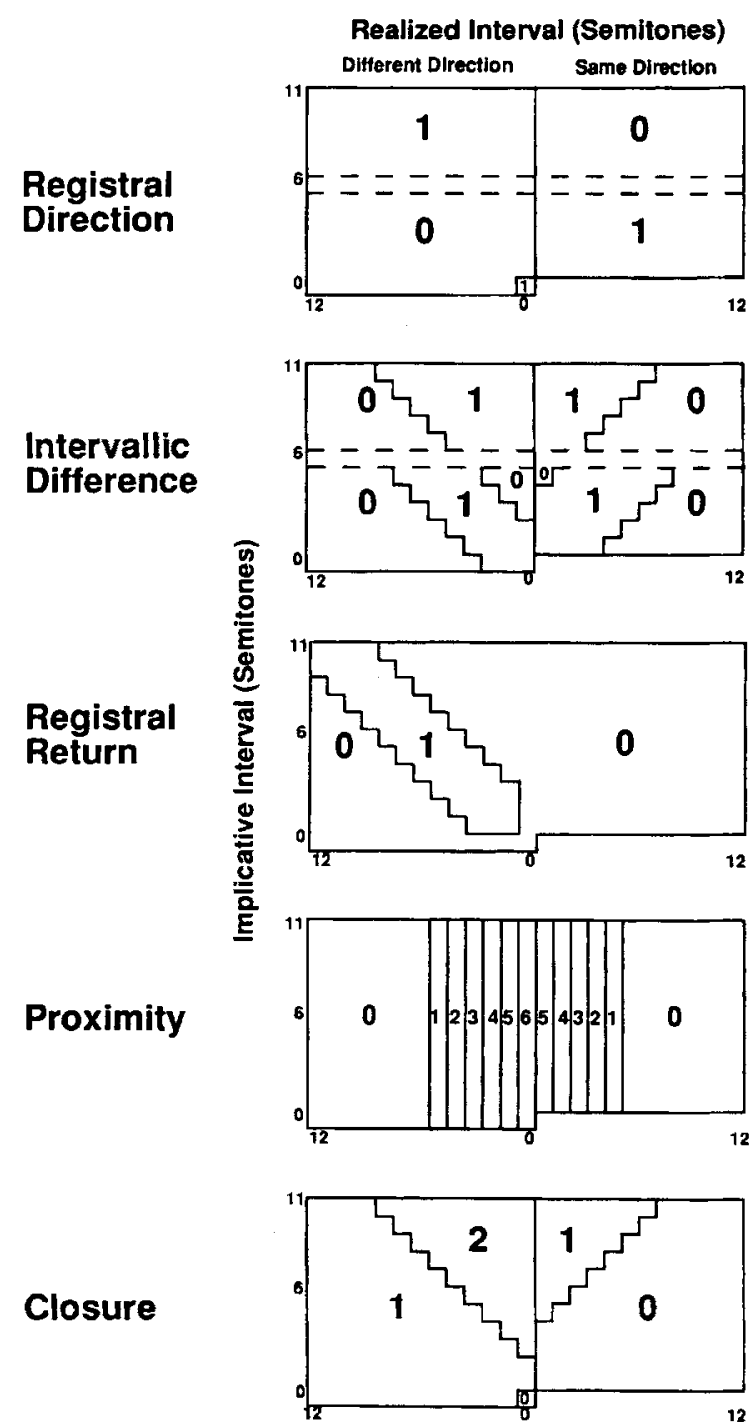

Figure 1. Narmour's (1990) bottom-up principles as quantified by Krumhansl (1995). From 'Music Psychology: Influences From Music Theory," by C. L. Krumhansl, 1995, Music Theory Spectrum, 17,53-80. Copyright 1995 by the Society for Music Theory. Reprinted with permission.

located within the chart. If the point of intersection falls in an area containing a nonzero code, the expectancy generated by the principle is said to be fulfilled. (Note that for the principles of proximity and closure there are graded values of fulfillment.) For the example, $\mathrm{C}_{4}-\mathrm{Eb}_{4}-$ $\mathrm{F}_{4}$, the expectancies generated by registral direction, intervallic difference, and proximity are fulfilled.

Alternatively, consider the implicative interval $\mathrm{Eb}_{4}-$ $\mathrm{C}_{5}$. This is an interval of nine semitones, called a major sixth, and it is considered a "large" interval. If it is followed by the note $\mathrm{F}_{4}$, the size of the realized interval, $\mathrm{C}_{5}-$ $\mathrm{F}_{4}$, is seven semitones (a perfect fifth), and the pitch direction is different from that of the implicative interval. Inspection of the charts in Figure 1 reveals that, for this 
second example, $\mathrm{Eb}_{4}-\mathrm{C}_{5}-\mathrm{F}_{4}$, the expectancies generated by registral direction, registral return, and closure are fulfilled.

There are three main predictions that can be made if Narmour's bottom-up principles are influencing melodic expectancies on a tone-to-tone level. The first is that the pattern of expectancies should depend on the size of the preceding implicative interval. Second, any given implicative interval should generate expectancies for a set of continuations (rather than one specific continuation), since different expectancies are generated by each principle. Finally, since these principles are assumed to be independent of style and musical experience, there should be no differences in their operation as a function of music training or exposure.

\section{Experimental Investigations With Melodic Beginnings}

Narmour (1990) acknowledged that there are situations in which both top-down and bottom-up processes might be operative, and situations in which one process might dominate the other. In ambiguous situations, such as at the beginning of melodies, he proposed that bottomup processes would dominate. Thus, to evaluate bottomup principles in accordance with Narmour's model, it is appropriate to start with the study of continuations for melodic beginnings.

Carlsen (1981) and Unyk and Carlsen (1987) investigated the melodic expectancies generated by melodic beginnings. Carlsen (1981) tested subjects from Germany, Hungary, and the United States who were in either an early or an advanced stage of professional music training. The subjects were presented with 25 melodic intervals - the 12 ascending and 12 descending equaltempered intervals within the octave, plus the unison. They were asked to consider each interval as the beginning of a melody and were instructed to sing the expected continuation of the melodic beginning. To determine the subjects' expectancies generated by the melodic beginning, Carlsen (1981) examined the interval between the second tone of the melodic beginning and the first tone of the sung response. The study by Unyk and Carlsen (1987) included a partial replication of Carlsen (1981) with U.S. music majors and faculty.

In both studies, it was found that different melodic beginnings generated different expectancies of varying strengths, as measured by the rank ordering of frequency of occurrence for different continuations. This finding is consistent with Narmour's (1990) assertion that different implicative intervals generate different patterns of expected continuations. There was also an overall tendency for subjects to produce small continuation intervals, as predicted by Narmour's proximity principle. Carlsen (1981) noted an absence of significant differences across the two levels of training. This absence supports the idea that certain aspects of melodic expectancy are not dependent on experience (although it should be noted that both groups were highly trained). Certain differences between different cultural groups were reported by Carlsen
(1981). The overall agreement among subjects in the two studies, however, was considerable, and it suggests the presence of common principles guiding, at least in part, judgments of melodic expectancy. Carlsen (1981) urged the collection of a general music expectancy database so that theories of expectancy could be empirically tested.

The present study was designed to explore the implication-realization model for melodic expectancy. As in the procedure of Carlsen (1981) and Unyk and Carlsen (1987), melodic intervals were used as the stimulus context - that is, as melodic beginnings. However, a perceptual task rather than a production task was employed. Perceptual tasks are a particularly important complement to production tasks in musical expectancy research. In music research, production tasks usually restrict the choice of subject population to trained performers (for further discussion, see Schmuckler, 1989, 1990), whereas perceptual tasks can be employed with novice listeners. Moreover, Narmour (1990) provides predictions for the relative strengths of all possible continuations, not just those that are likely to be sung. Therefore, we selected a perceptual rather than a production task in order to evaluate the strength of each of an exhaustive set of continuations over the range of two octaves.

Eight melodic intervals (two small and two large intervals, in both ascending and descending order) were presented to both musically trained and untrained listeners. They were asked to consider each interval as the beginning of a melody, and to rate how well each of the possible chromatic tones within an octave above and below the second note of the interval continued the melody. There was, therefore, an orthogonal combination of eight implicative intervals and 25 realized intervals-yielding a set of 200 three-note patterns. These patterns provided instances of both the fulfillment and the nonfulfillment of expectancies for each of the five bottom-up principles illustrated in Figure 1.

The continuation ratings were considered a measure of the extent to which the expectancies generated by the implicative interval was fulfilled by the realized interval. The notion of continuation is central to Narmour's $(1989,1990,1991)$ proposals; "melody frequently generates conscious and subconscious expectations as to what continuation seems most probable" (1989, p. 46). In such a minimal context as that tested here, judgments of continuity should reveal the influence of bottom-up factors such as those proposed by the implicationrealization model.

\section{METHOD}

\section{Subjects}

Twenty-four undergraduate students at Queen's University participated in this study. Twelve musically trained subjects ( $9 \mathrm{fe}-$ males and 3 males) were recruited through a subject pool at the School of Music at Queen's University. Music training was defined as Grade IX Royal Conservatory of Music training or enrollment in the 2nd year or higher of the Bachelor of Music program. These musically trained subjects ranged in age from 19 to 
22 with a mean age of 20.8 years, and they had a mean of 13.2 years of musical training $(S D=2.4)$. They were paid $\$ 7$ for their participation. Twelve musically untrained subjects ( 10 females and 2 males) were recruited through the Introductory Psychology Subject Pool at Queen's University. Musically untrained subjects ranged in age from 18 to 22 with a mean age of 19.4 years, and had a mean of 1.9 years of musical training $(S D=1.5)$. They were given course credit for their participation.

\section{Stimulus Materials}

Eight intervals (four interval sizes $\times$ two directions of presentation) were chosen as representative of small and large intervals as defined by the implication-realization model. These intervals were the implicative intervals for the experiment. The small intervals consisted of a major second ( 2 semitones) and a minor third (3 semitones). The large intervals consisted of a major sixth (9 semitones) and a minor seventh (10 semitones). Each interval was presented in its ascending and descending form. In addition, two versions of each of the eight intervals were created: one for which the second tone of the interval was $\mathrm{C} 4$, and one for which the second tone was $\mathrm{F}_{4}$. The notes $\mathrm{C}$ and $\mathrm{F} \#$ represent two highly unrelated keynotes (Krumhansl, 1990). The presence of unrelated keynotes was intended to discourage subjects from developing a top-down sense of tonality for the entire experiment. Table 2 lists all the intervals used as implicative intervals.

The implicative interval was presented as a long-short duration pattern (dotted half-note followed by a quarter note) and a strongweak accent pattern. Specifically, the duration of the first tone, $1.2 \mathrm{sec}$, was three times as long as that of the second tone, $0.4 \mathrm{sec}$, and the amplitude of the first tone was $40 \%$ greater than that of the second. These manipulations were intended to create a sense of $4 / 4$ meter that would continue from the first measure (containing the implicative interval) to the next measure (containing the continuation tone). Continuation tones consisted of all 25 tones from one octave below to one octave above the second tone of the implicative interval (from either $\mathrm{C}_{3}$ to $\mathrm{C}_{5}$ or $F \#_{3}$ to $\mathrm{FH}_{5}$ ). Each continuation tone was $0.8 \mathrm{sec}$ in duration and was equal in amplitude to the second tone of the implicative interval. The total duration of each three-tone pattern was $2.4 \mathrm{sec}$. All stimulus materials were created with the Cakewalk Professional software for Windows (Hendershott, 1990).

\section{Apparatus}

Subjects were tested in a soundproof booth in the Acoustical Laboratory of Queen's University. Presentation of tones and collection of responses were controlled by a Zenith Z-200 computer running software written by Fahir Ergincan. Tones were generated by a Yamaha TX-802 synthesizer, set to "wood piano," and connected to the Z-200 computer through a Roland MPU-401 interface. Subjects heard the trials through Monitor Audio R252 speak-

Table 2

Large and Small Implicative Intervals Ending on $\mathrm{C}$ and $\mathrm{F}$ \#

\begin{tabular}{|c|c|c|c|}
\hline \multirow[b]{2}{*}{ Interval } & \multirow[b]{2}{*}{ Direction } & \multicolumn{2}{|c|}{ Second Note } \\
\hline & & $\mathrm{C}$ & $F^{\#}$ \\
\hline Major second & $\begin{array}{l}\text { Ascending } \\
\text { Descending }\end{array}$ & $\begin{array}{l}\mathrm{Bb}_{3}-\mathrm{C}_{4} \\
\mathrm{D}_{4}-\mathrm{C}_{4}\end{array}$ & $\begin{array}{r}\mathrm{E}_{4}-\mathrm{F}_{4} \\
\mathrm{G}_{4} \mathbb{H}_{4}-\mathrm{F}_{4}^{\#_{4}}\end{array}$ \\
\hline Minor third & $\begin{array}{l}\text { Ascending } \\
\text { Descending }\end{array}$ & $\begin{array}{c}\mathrm{A}_{3}-\mathrm{C}_{4} \\
\mathrm{~Eb}_{4}-\mathrm{C}_{4}\end{array}$ & $\begin{array}{r}\mathrm{D} \#_{4}-\mathrm{F}_{4} \\
\mathrm{~A}_{4}-\mathrm{F}_{4}\end{array}$ \\
\hline Major sixth & $\begin{array}{l}\text { Ascending } \\
\text { Descending }\end{array}$ & $\begin{array}{r}\mathrm{E}_{3}-\mathrm{C}_{4} \\
\mathrm{~A}_{4}-\mathrm{C}_{4}\end{array}$ & $\begin{array}{r}\mathrm{A}_{3}-\mathrm{F}_{4} \\
\mathrm{D} \#_{5}-\mathrm{F}_{4}\end{array}$ \\
\hline Minor seventh & $\begin{array}{l}\text { Ascending } \\
\text { Descending }\end{array}$ & $\begin{array}{l}\mathrm{D}_{3}-\mathrm{C}_{4} \\
\mathrm{Bb}_{4}-\mathrm{C}_{4}\end{array}$ & $\begin{array}{r}\mathrm{G}_{3}-\mathrm{F}_{4} \\
\mathrm{E}_{5}-\mathrm{F}_{4} \\
\end{array}$ \\
\hline
\end{tabular}

ers at a comfortable loudness level (approximately 60-65 dB SPL).

\section{Procedure}

After having consented to participate in the experiment, the subjects were seated in front of a terminal in the soundproof booth. Instructions were given both verbally and presented on the computer screen. The subjects were told that, on each trial, they would hear a two-note pattern which they were to consider as the beginning of a melody. They were then told that this melodic beginning would be followed by a test tone, and that they were to rate how well the test tone continued the melody on a scale from 1 (extremely bad continuation) to 7 (extremely good continuation). It was stressed that they should try to use the full range of possible ratings. The subjects were told that there would be eight different melodic beginnings and a series of test tones for each beginning. A practice interval (not used in the rest of the investigation) was presented with five different continuation tones in order to familiarize the subjects with the task, after which any additional questions were addressed.

The experiment consisted of eight blocks of 27 trials each. The first 2 trials of each block were considered practice and were not considered in the data analyses. One of the implicative intervals was tested in each block. Each trial in each block consisted of the implicative interval followed by a randomly selected continuation tone, after which the subjects were prompted by the computer to rate how well the tone continued the melodic beginning. On subsequent trials of a block, the same implicative interval was followed by a different continuation tone selected randomly without replacement from the set of 25 continuation tones. The pace of presentation was determined by the subject.

The order of presentation of the blocks (implicative intervals) was randomized for each subject with the constraint that no consecutive blocks contain implicative intervals that started or ended on the same note. Each subject heard half of the implicative intervals ending on $\mathrm{C}_{4}$ and half ending on $\mathrm{F}_{4}$ (in an attempt, as noted above, to discourage a key bias). After completing all 200 trials, the subjects were informed of the purpose of the study. Each experimental session lasted approximately $50 \mathrm{~min}$.

\section{RESULTS}

The collected data yielded 200 continuation ratings for each subject -25 ratings for each of eight implicative intervals. The mean continuation rating for each continuation tone for each implicative interval, calculated separately for the musically trained and the untrained listeners, is given in the Appendix. The correlation between mean continuation ratings for trained and untrained subjects was $.88(d f=198, p<.001)$, indicating a high degree of similarity in responses for the two groups.

To determine the extent of intersubject agreement, the 200 continuation ratings for each subject were correlated with those of every other subject. The overall mean intersubject correlation coefficient was .38 , with a range of -.05 to .71 . Of the 276 pairwise intersubject correlations, all but 14 were statistically significant $(d f=198$, with at least $p<.05$ ). Of these 14 nonsignificant correlations, 10 involved a single trained subject. (This subject was not, however, excluded from the subsequent data analyses, because there was no apparent reason to 
reject the subject.) The mean intersubject correlation for musically trained subjects was .41 , with a range of -.01 to .59 . The mean intersubject correlation for musically untrained subjects was .35 , with a range of .06 to .62 .

A three-factor mixed analysis of variance (ANOVA) was conducted on the continuation ratings (Keppel, 1991). The between-subjects factor was musical training with 2 levels, and the within-subjects factors were continuation tone with 25 levels and implicative interval with 8 levels. The ANOVA was conducted as a further check, preliminary to model testing, that differences among continuation ratings were reliable for both training groups.

There was a significant main effect of continuation tone $[F(24,528)=39.73, p<.0001]$, a result indicating that there were differences in the mean ratings for each of the 25 continuations collapsed across the melodic beginnings. As expected, there was only one significant interaction, continuation tone $\times$ implicative interval $[F(168,3696)=$ $7.14, p<.0001]$, a result indicating that the ratings assigned to the 25 continuation tones depended on the type of preceding implicative interval. The lack of significant interactions with training indicates, as does the correlation between training levels reported above, a strong correspondence between the responses of the musically trained and the untrained subjects.

Although the interaction between continuation tone and implicative interval was significant, as predicted by the implication-realization model, the interaction does not necessarily reflect the influence of the operation of Narmour's $(1990,1991)$ bottom-up principles. A series of models was therefore examined, and each model was tested through multiple regression analysis. The first model assessed the five bottom-up principles of the implication-realization model. Subsequent models assessed other factors to account for variance in the data, including factors reflecting top-down processes of tonal organization. Because no significant differences were found between trained and untrained subjects and the degree of intersubject agreement was high, the 25 continuation ratings for each interval were averaged across subjects and levels of training. The dependent variable for the regression analyses was a vector of 200 ratings corresponding to the mean continuation ratings for each combination of continuation tone and implicative interval.

The first multiple regression analysis is summarized in Table 3. The five bottom-up principles of the implication-realization model (registral direction, intervallic difference, registral return, proximity, and closure) were entered as predictors of the averaged continuation ratings. These predictors were quantified according to the charts in Figure 1. The fit of the model to the data was highly significant, accounting for approximately $29 \%$ of the variance in the mean ratings $(R=.54, p<.0001)$. Of the five principles entered, the beta weights for registral return and proximity were both significant, at the $p<$ .01 and $p<.001$ levels, respectively, and the beta weights for intervallic difference, registral direction, and closure
Table 3

A Summary of the Multiple Regression Analysis With Narmour's (1990) Five Bottom-Up Principles

\begin{tabular}{llll}
\hline \multicolumn{1}{c}{ Predictor } & $\boldsymbol{\beta}$ & $t$ & $p$ \\
\hline Registral direction & .09 & 1.52 & $<.14$ \\
Intervallic difference & .17 & 1.83 & $<.07$ \\
Registral return & .16 & 2.61 & $<.01$ \\
Proximity & .30 & 3.40 & $<.001$ \\
Closure & .12 & 1.71 & $<.09$ \\
\hline
\end{tabular}

Note-Overall model: $R=.54 ; F(5,194)=15.57, p<.0001$.

approached significance. The predictors of intervallic difference and registral direction were significant in subsequent analyses, but the predictor of closure was not. Closure will be dropped from further discussion.

In the next step of model testing, four principles were retained, but the predictor of registral direction was modified. Examination of the residual variance revealed that registral direction seemed to be operating for large intervals only. A new predictor was created, which contained zeros for the small intervals and the original predictions for the large intervals. A predictor named pitch height was created, based on the observation that ratings tended to increase as the pitch height of the continuation tone increased. For the pitch height predictor, continuation tones were ranked in order from 1 (for the lowest continuation tone) to 25 (for the highest continuation tone). The lowest continuation tone was one octave below the second tone of the implicative interval, and the highest continuation tone was one octave above the second tone of the implicative interval.

Tonality predictors were also included at this step. The tonality predictors tested the assumption that each tone of the implicative interval was perceived as a keynote or tonic. If so, continuity ratings could be based, in part, on the degree to which the continuation tone fit the tonal hierarchy of the tonic note. Four tonal hierarchy predictors were constructed: (1) the tonal hierarchy of the major key for the first note of the implicative interval; (2) the tonal hierarchy of the major key for the second note; (3) the tonal hierarchy of the minor key for the first note; and (4) the tonal hierarchy of the minor key for the second note. The entries for these tonal hierarchy predictors were based on the quantification of the tonal

Table 4

A Summary of the Multiple Regression Analysis With Revised Narmour (1990) Principles, Pitch Height, and Tonal Hierarchy Predictors

\begin{tabular}{llll}
\hline \multicolumn{1}{c}{ Predictor } & $\boldsymbol{\beta}$ & $t$ & $p$ \\
\hline Registral direction (revised) & .11 & 1.98 & $<.05$ \\
Intervallic difference & .29 & 3.70 & $<.001$ \\
Registral return & .16 & 2.99 & $<.01$ \\
Proximity & .24 & 3.20 & $<.01$ \\
Pitch height & .26 & 5.09 & $<.0001$ \\
Major tonal hierarchy, 1st note & .10 & 1.62 & $<.11$ \\
Major tonal hierarchy, 2nd note & .16 & 2.52 & $<.02$ \\
Minor tonal hierarchy, 1st note & .22 & 3.77 & $<.001$ \\
Minor tonal hierarchy, 2nd note & .16 & 2.36 & $<.02$ \\
\hline
\end{tabular}

Note-Overall model: $R=.70 ; F(9,190)=20.75, p<.0001$. 
hierarchy collected by Krumhansl and Kessler (1982)the values are available in Krumhansl (1990, p. 37).

A summary of the analysis is contained in Table 4. This model accounted for $49 \%$ of the variance in subjects' averaged ratings $(R=.70, p<.0001)$. All of the beta weights for the predictors were significant, with the exception of the beta weight for the first tonality predictor $(p<.11)$.

The next stage of the model testing involved the creation of a second type of tonality predictor. The tonality predictors in the previous model were derived from the assumption that the individual tones of each implicative interval instantiated the tonal hierarchy of the keys for which these tones were the tonic notes. It is also possible that the pattern of the three tones presented on each trial conveyed a sense of tonal relationship. If this is so, the strength of the tonal relationship would vary with the particular combination of implicative interval and continuation tone. A predictor named tonal strength was derived from the assumption that the rating of a continuation tone would be influenced by the degree to which the pattern of three tones suggested a tonality.

Using a key-finding algorithm developed by Krumhansl and Schmuckler (in Krumhansl, 1990), input vectors containing duration and pitch values for each of the 200 three-note patterns were correlated with tonal hierarchy vectors for every major and minor key. The highest significant correlation (with at least $p<.10$ ) between the input vector and the tonal hierarchy vectors for each three-note pattern was used to create a tonal strength predictor. A zero was entered for a pattern if there were no significant correlations for that pattern. Thus, the tonal strength predictor was a measure of the extent to which the three notes on a given trial "fit together" tonally. This predictor, added to the model above, was significant, and the amount of variance accounted for was incremented $(R=.73, p<.0001)$. The analysis is summarized in Table 5.

Even with tonal strength added to the regression, almost half of the variance was still unaccounted for. A third type of tonality predictor was explored. Here it was assumed that, because each implicative interval could

Table 5

A Summary of the Multiple Regression Analysis With Revised Narmour (1990) Principles, Pitch Height, Tonal Hierarchy Predictors, and Tonal Strength Predictor

\begin{tabular}{lccc}
\hline \multicolumn{1}{c}{ Predictor } & $\beta$ & $t$ & $p$ \\
\hline Registral direction (revised) & .09 & 1.75 & $<.09$ \\
Intervallic difference & .27 & 3.66 & $<.001$ \\
Registral return & .22 & 4.05 & $<.001$ \\
Proximity & .27 & 3.67 & $<.001$ \\
Pitch height & .26 & 5.17 & $<.0001$ \\
Major tonal hierarchy, 1st note & .03 & 0.47 & $<.65$ \\
Major tonal hierarchy, 2nd note & .14 & 2.23 & $<.03$ \\
Minor tonal hierarchy, 1st note & .13 & 2.20 & $<.03$ \\
Minor tonal hierarchy, 2nd note & .12 & 1.94 & $<.06$ \\
Tonal strength & .25 & 4.08 & $<.001$ \\
\hline Note-Overall model: $R=.73 ; F(10,189)=21.88, p<.0001$.
\end{tabular}

Table 6

A Summary of the Multiple Regression Analysis With Revised Narmour (1990) Principles, Pitch Height, Tonal Strength Predictor, and Tonal Region

\begin{tabular}{lccc}
\hline \multicolumn{1}{c}{ Predictor } & $\beta$ & $t$ & $p$ \\
\hline Registral direction (revised) & .11 & 2.47 & $<.02$ \\
Intervallic difference & .25 & 3.94 & $<.001$ \\
Registral return & .18 & 3.82 & $<.001$ \\
Proximity & .28 & 4.49 & $<.0001$ \\
Pitch height & .28 & 6.49 & $<.0001$ \\
Tonal strength & .13 & 2.53 & $<.02$ \\
Tonal region & .47 & 9.52 & $<.0001$ \\
\hline
\end{tabular}

Note-Overall model: $R=.80 ; F(7,192)=45.85, p<.0001$

belong to several keys, a number of different major and minor keys might be weakly implicated by the interval. This assumption differs from the assumption underlying the tonal hierarchy predictors of previous models, which are based on the specific hierarchy of the key of one or both of the notes of the implicative interval.

The quantification of the third type of tonality predictor was suggested by Krumhansl (personal communication, June 18, 1993). First, all possible major and (natural) minor keys in which each implicative interval could occur were listed. Next, each continuation tone was coded according to whether it was a possible tonic for the preceding interval. That is, the continuation tones that were the tonic of major or natural minor keys that contained the two notes of the implicative interval were coded as " 1 " and all other continuations were coded as "0." This predictor was named tonal region, because it suggests that a region, or subset, of keynotes is instantiated by the implicative interval rather than the specific hierarchies of the notes contained in the interval.

A final multiple regression was conducted and is summarized in Table 6. In this analysis, the predictors entered were the four bottom-up predictors from Table 4, plus pitch height, tonal strength, and tonal region. (In an intervening step, it was found that the tonal hierarchy predictors of Table 4 could be dropped without any significant loss in prediction.) The overall model accounted for $64 \%$ of the variance in mean continuation ratings $(R=.80, p<.0001)$. All seven beta weights for the predictors were significant at the .001 level.

As a measure of consistency, this final model was then fit to the continuation ratings for each individual subject. The overall model provided a significant fit to the data for each subject (at the $p<.0001$ level for 23 subjects, and the $p<.008$ level for the trained subject with the low intersubject correlations), with a mean $R=$ .60 (range of $R=.31$ to .79 ). Of the predictors, the beta weight for the tonal region predictor was most consistently significant (for 22 of the 24 subjects, $p<.05$ ), followed by pitch height (for 16 of the subjects), registral return and proximity (for 13 of the subjects), intervallic difference (for 9 of the subjects), registral direction (for 7 of the subjects), and tonal strength (for 6 of the subjects). The mean beta weight for each predictor in the 
final model was found to be significantly different from zero; this analysis is summarized in Table 7.

As a final check for any differences between the musically trained and untrained groups, the model was also tested against the mean ratings separately for the two groups. The fit of the model was highly significant for both groups (for the musically trained group, $R=.75$, $p<.0001$; for the untrained group, $R=.81, p<.0001$ ), and all of the beta weights for each predictor were significant. The two solutions were, in fact, virtually identical to the overall model presented in Table 6-a not surprising result, given the high degree of agreement between the data for the two training groups. Thus both the influences of the bottom-up principles, as revised in the final model, and the tonal predictors were revealed for both groups.

The next analyses involved examining the relation between the mean continuation ratings of the present study and the production data collected by Carlsen (1981). The Carlsen (1981) data consist of the frequency of occurrence-or, equivalently, percentage - of tones sung by 91 musically trained persons as continuations of each of 25 two-note melodic patterns. The rank-order correlation between the present data and the Carlsen data for the implicative intervals tested here was $.66(d f=198$, $p<.001$ ).

The ranked Carlsen data were regressed on the five bottom-up principles as proposed by Narmour (1990; Table 3 ) and the predictors for the final model for the present data (Table 6). The model based on the five bottomup principles provided an excellent fit to the data $(R=$ $.85, p<.0001)$. Beta weights for all predictors were significant. The final model provided a slightly better fit ( $R=.87, p<.0001)$. The bottom-up principles, as entered in the final model, were significant predictors. Pitch height was not significant. The tonality predictors of the final model were significant and added a slight increment in the variance accounted for beyond that accounted for by the Narmour principles of the final model.

It may be concluded that there is good agreement between the present perception data and the Carlsen (1981) production data. With some caution, because of the differences in the dependent measures, it may be tentatively concluded that the influence of the bottom-up principles was somewhat more evident in the production data, and the influence of the tonality predictors was somewhat more evident in the perception data.

Table 7

A Summary of the $t$ Tests Comparing the Mean $\beta$ Weights of the Final Model for All 24 Subjects to Zero

\begin{tabular}{lccc}
\hline \multicolumn{1}{c}{ Predictor } & Mean $\boldsymbol{\beta}$ Weight & $t$ & $p$ \\
\hline Registral direction (revised) & .07 & 4.68 & $<.001$ \\
Intervallic difference & .16 & 7.34 & $<.001$ \\
Registral return & .11 & 5.92 & $<.001$ \\
Proximity & .17 & 5.33 & $<.001$ \\
Pitch height & .18 & 4.45 & $<.001$ \\
Tonal strength & .08 & 4.86 & $<.001$ \\
Tonal region & .30 & 9.50 & $<.001$ \\
\hline
\end{tabular}

\section{DISCUSSION}

In this study, we evaluated the five bottom-up principles of Narmour's (1990) music-theoretic model of implication-realization as predictors of listener's melodic expectancies. Quantified predictors were tested against continuity ratings collected for a tone that followed a two-tone melodic beginning. A series of regression analyses was conducted on the ratings, beginning with the test of the bottom-up principles. Subsequent regression analyses examined additional influences on the continuity ratings and included a test of quantified predictors based on the Western tonal-harmonic idiom.

There were three main findings. First, there was support for three of Narmour's (1990) five principles of toneto-tone melodic expectancy-intervallic difference, registral return, and proximity - and support for a modified version of a fourth principle - registral direction. Second, there was support for Narmour's (1990) assertion that the operation of the bottom-up principles of expectancy is independent of experience, at least for the range of experience tested here. Third, there was evidence that, in addition to the principles proposed by the implication-realization model, there was a strong influence of perceived tonality on continuation judgments. This influence-as quantified by the predictors of tonal strength and tonal region-was also not specific to formal musical training.

Despite the support that was found for the implicationrealization model, the present findings also raise some questions about its present formulation. First, the predictor of registral direction was operative for large intervals only. Second, there was no support for the influence of principle of closure. It was also noted that pitch height accounted for a significant amount of variance in continuity ratings. Although this is not a predictor in Narmour's (1990) model, it might also be considered as a bottom-up influence on melodic expectancy.

These findings urge several lines of further work. One line of inquiry might be to ascertain the degree to which the present findings are attributable to the simple, abstract paradigm employed here. The principle of closure, for example, may not exert an influence on expectancies for the third tone of a melody, but might exert considerable influence within the richer context of a full melody. A melodic opening may, as Narmour (1990) has suggested, be an instance in which bottom-up principles should dominate; this was the reason for the selection of the present paradigm. Alternatively, however, it may be the case that the influence of the principles becomes increasingly apparent as a melody unfolds. A further problem here for future research concerns the nonorthogonality of the principles, and the possible suppression of one by another in specific contexts. Possibly alternative formulations should be considered to circumvent this problem (Krumhansl, 1995).

Two constraints on the interpretations of the findings may also be mentioned. First, the experiment was not intended to address whether the bottom-up principles 
reflect innate perceptual properties. Casual (as opposed to formal) exposure to the idiom of the Western culture may have played a role in the correspondence between the judgments of the two training groups for both bottom-up and tonal predictors. Developmental, and cross-cultural, studies may be usefully initiated to address these issues.

Second, the experiment was not intended to address the role of the top-down processes to which patterns within musical pieces are subjected (Narmour, 1990). We leave to future work the question of how bottom-up and top-down processing may function in opposition, and the question of how the bottom-up principles of continuation may be violated, in actual music, for aesthetic effect.

Significant correspondence was found between the continuation judgments in the present study and the production data collected for the same implicative intervals by Carlsen (1981). This correspondence supports arguments by Palmer (1992) and Schmuckler $(1989,1990)$ that music perception and performance have common strategies. In particular, our data support conclusions by Schmuckler (1989) that there is converging evidence for expectancy generation in the two tasks.

Finally, it is interesting to note the large proportion of variance in subjects' continuation ratings that could be attributed to tonal influences. The fact that the influence of tonality was similar across musically trained and untrained subjects is consistent with recent evidence (e.g., Cuddy \& Badertscher, 1987; Steinke, Cuddy, \& Holden, 1993) that musically untrained subjects are able to recover the hierarchy of tonal function.

An important contribution of the present research is the construction of the tonal region predictor, which defines a set of equally possible keys that might be implied in an ambiguous context. The simple experimental contexts used here were ambiguous with respect to key, but, in addition, there are also "real-life" musical situations in which no clear key is implied. In these cases, no one tonal hierarchy would be useful in understanding musical structure. The fact that this predictor was an important influence on subjects' judgments of continuations suggests that listeners are flexible enough to change to a less specific and perhaps more helpful strategy when necessary. Continuation ratings for other implicative intervals should be collected to determine the general applicability of the tonal region predictor.

Psychological investigations of Narmour's (1990) implication-realization model are important for a number of reasons. Unlike many other Gestalt-based theories, its principles are precisely stated and empirically testable. Despite the limitations discussed above, this study furthers our knowledge about the applicability of the implicationrealization model to melodic expectancy (an endeavour that Narmour invites). In addition to melody, Narmour (1990) has proposed that his model applies to musical parameters such as rhythm and the perception of musical form. Meyer's (1956) theory dealt with the experience of emotion as a result of unrealized expectancies and their resolutions, suggesting the possibility of extending the principles of the implication-realization theory to explain affective responses to music. Ultimately, therefore, Narmour's (1990) model promises wide applicability to naturalistic contexts of music listening.

As Krumhansl (1995) points out, music-theoretic concepts are not often subjected to empirical tests. It is rare that music theorists are interested in making their theories and concepts empirically testable. Music theorists and psychologists sometimes seem to speak a different language, and, even when they do use the same terms, they are often using a different dictionary. Nevertheless, the insights and intuitions of contemporary music theory are powerful guides to understanding complex auditory processing. Studies which investigate the limits and validity of music theories are essential to bridge the gap between the disciplines of music theory and music perception.

\section{REFERENCES}

ANDERSON, J. R. (1990). Cognitive psychology and its implications (3rd ed.). New York: W. H. Freeman.

AUBEL, P., \& FRANKS, J. J. (1983). Sentence comprehension processes. Journal of Verbal Learning \& Verbal Behavior, 22, 395-405.

BERRY, W. (1976). Structural functions in music. Englewood Cliffs, NJ: Prentice-Hall.

Boltz, M. G. (1993). The generation of temporal and melodic expectancies during musical listening. Perception \& Psychophysics, 53, 585-600.

BRANSFORD, J. D., \& Johnson, M. K. (1972). Conceptual prerequisites for understanding. Journal of Verbal Learning \& Verbal Behaviour, 11, 717-721.

Bregman, A. S. (1990). Auditory scene analysis. Cambridge, MA: MIT Press.

CARlsen, J. C. (1981). Some factors which influence melodic expectancy. Psychomusicology, 1, 12-29.

Carlsen, J. C. (1990). Preface. Psychomusicology, 9, 119-121.

Coren, S., Porac, C., \& TheODor, L. H. (1986). The effects of perceptual set on the shape and apparent depth of subjective contours. Perception \& Psychophysics, 39, 327-333.

CUDDY, L. L., \& BADERTSCHER, B. (1987). Recovery of the tonal hierarchy: Some comparisons across age and levels of musical experience. Perception \& Psychophysics, 41, 609-620.

Deutsch, D. (1982). Grouping mechanisms in music. In D. Deutsch (Ed.), The psychology of music (pp. 99-134). New York: Academic Press.

Dodwell, P. C. (1993). From the top down. Canadian Psychology, 34, 137-151

Dowling, W. J. (1990). Expectancy and attention in melody perception. Psychomusicology, 9, 148-160.

Dowling, W. J., \& HaRWood, D. L. (1986). Music cognition. Orlando, FL: Academic Press.

GarNer, W. R. (1981). The analysis of unanalyzed perceptions. In M. Kubovy \& J. R. Pomerantz (Eds.), Perceptual organization (pp. 119-140). Hillsdale, NJ: Erlbaum.

Hendershott, G. (1990). Cakewalk Professional for Windows [Computer program]. Watertown, MA: Twelve Tone Systems.

JONES, M. R. (1981). Music as a stimulus for psychological motion: Part I. Some determinants of expectancies. Psychomusicology, 1 , 34-51.

Jones, M. R. (1982). Music as a stimulus for psychological motion: Part II. An expectancy model. Psychomusicology, 2, 1-13.

JoNEs, M. R. (1990). Learning and the development of expectancies: An interactionist approach. Psychomusicology, 9, 193-228. 
JONES, M. R., \& Boltz, M. G. (1989). Dynamic attending and responses to time. Psychological Review', 96, 459-491.

KEPPEL, G. (1991). Design and analysis: A researcher's handbook. Englewood Cliffs, NJ: Prentice-Hall.

KRUMHANSL, C. L. (1990). Cognitive foundations of musical pitch. New York: Oxford University Press.

KRUMHANSL, C. L. (1991). Melodic structure: Theoretical and empirical descriptions. In J. Sundberg, L. Nord, \& R. Carlson (Eds.), Music, language, speech and brain (pp. 269-283). London: Macmillan.

KrumhanSL, C. L. (1995). Music psychology: Influences from music theory. Music Theory Spectrum, 17, 53-80.

Krumhansl, C. L., \& Kessler, E. J. (1982). Tracing the dynamic changes in perceived tonal organization in a spatial representation of musical keys. Psychological Review, 89, 334-368.

MEYer, L. B. (1956). Emotion and meaning in music. Chicago: University of Chicago Press.

MiLls, C. B. (1980). Effects of the match between listener expectancies and coarticulatory cues on the perception of speech. Journal of Experimental Psychology: Human Perception \& Performance, 6, 528-535.

Narmour, E. (1989). The "genetic code" of melody: Cognitive structures generated by the implication-realization model. Contemporary Music Review, 4, 45-63.

NARMOUR, E. (1990). The analysis and cognition of basic melodic structures: The implication-realization model. Chicago: University of Chicago Press.
Narmour, E. (1991). The top-down and bottom-up systems of musical implication: Building on Meyer's theory of emotional syntax Music Perception, 9, 1-26.

PALMer, C. (1992). The role of interpretive preferences in music performance. In M. R. Jones \& S. Holleran (Eds.), Cognitive bases of musical communication (pp. 249-262). Washington, DC: American Psychological Association.

Palmer, C., \& Krumhansl, C. L. (1990). Mental representations for musical meter. Journal of Experimental Psychology: Human Perception \& Performance, 16, 728-741.

Pomerantz, J. R. ( 1981 ). Perceptual organization in information processing. In M. Kubovy \& J. R. Pomerantz (Eds.), Perceptual organization (pp. 141-180). Hillsdale, NJ: Erlbaum.

Schiffman, H. R. (1990). Sensation and perception: An integrated approach (3rd ed.). New York: Wiley.

SCHMUCKLER, M. A. (1989). Expectation in music: Investigation of melodic and harmonic processes. Music Perception, 7, 122147.

SCHMUCKLER, M. A. (1990). The performance of global expectations. Psychomusicology, 9, 122-147.

Steinke, W. R., Cuddy, L. L., \& Holden, R. R. (1993). Perception of musical tonality as assessed by the probe-tone method. Canadian Acoustics, 21, 85-86.

UNYK, A., \& CARLSEN, J. C. (1987). The influence of expectancy on melodic perception. Psychomusicology, 7, 3-23.

\section{APPENDIX}

The four tables of the Appendix provide the mean rating $(M)$ and standard error $(S E)$ for each continuation tone (C, ordered from lowest to highest) for each of the eight implicative intervals $(n=12)$. Ratings for each training level are provided separately. The correlation between training levels is also given for each implicative interval.

Table A1

Descriptive Statistics for Ratings of Continuation Tones Following the Implicative Interval of a Major Second

\begin{tabular}{|c|c|c|c|c|c|c|c|c|}
\hline \multirow[b]{3}{*}{$\mathrm{C}$} & \multicolumn{4}{|c|}{ Ascending } & \multicolumn{4}{|c|}{ Descending } \\
\hline & \multicolumn{2}{|c|}{ Trained } & \multicolumn{2}{|c|}{ Untrained } & \multicolumn{2}{|c|}{ Trained } & \multicolumn{2}{|c|}{ Untrained } \\
\hline & $M$ & $S E$ & $M$ & $S E$ & $M$ & $S E$ & $M$ & $S E$ \\
\hline 1 & 3.58 & 0.31 & 2.75 & 0.51 & 3.92 & 0.43 & 2.67 & 0.33 \\
\hline 2 & 2.08 & 0.36 & 2.25 & 0.30 & 1.75 & 0.33 & 1.83 & 0.24 \\
\hline 3 & 2.58 & 0.40 & 2.50 & 0.26 & 2.75 & 0.33 & 3.08 & 0.47 \\
\hline 4 & 3.83 & 0.59 & 4.08 & 0.47 & 2.42 & 0.19 & 2.75 & 0.30 \\
\hline 5 & 3.00 & 0.44 & 3.17 & 0.32 & 3.08 & 0.45 & 3.25 & 0.37 \\
\hline 6 & 5.33 & 0.45 & 5.58 & 0.26 & 5.33 & 0.31 & 4.33 & 0.43 \\
\hline 7 & 2.58 & 0.38 & 3.33 & 0.36 & 2.92 & 0.40 & 2.83 & 0.24 \\
\hline 8 & 4.17 & 0.34 & 4.83 & 0.37 & 4.58 & 0.47 & 5.00 & 0.35 \\
\hline 9 & 5.25 & 0.39 & 5.25 & 0.35 & 3.50 & 0.42 & 3.50 & 0.45 \\
\hline 10 & 4.92 & 0.43 & 4.83 & 0.42 & 5.50 & 0.26 & 4.83 & 0.32 \\
\hline 11 & 4.42 & 0.38 & 5.08 & 0.34 & 5.67 & 0.41 & 5.25 & 0.33 \\
\hline 12 & 3.42 & 0.34 & 2.92 & 0.45 & 4.75 & 0.45 & 4.42 & 0.53 \\
\hline 13 & 4.42 & 0.31 & 5.50 & 0.29 & 4.67 & 0.40 & 5.42 & 0.36 \\
\hline 14 & 6.00 & 0.28 & 5.08 & 0.34 & 3.50 & 0.47 & 3.83 & 0.47 \\
\hline 15 & 5.92 & 0.40 & 5.33 & 0.36 & 4.67 & 0.31 & 4.92 & 0.50 \\
\hline 16 & 5.75 & 0.33 & 5.67 & 0.33 & 5.17 & 0.41 & 5.92 & 0.36 \\
\hline 17 & 4.25 & 0.37 & 3.17 & 0.42 & 5.08 & 0.38 & 4.42 & 0.42 \\
\hline 18 & 5.42 & 0.43 & 5.08 & 0.23 & 6.25 & 0.35 & 5.17 & 0.27 \\
\hline 19 & 2.92 & 0.31 & 3.67 & 0.43 & 3.00 & 0.30 & 3.42 & 0.36 \\
\hline 20 & 4.00 & 0.35 & 5.25 & 0.49 & 5.83 & 0.24 & 5.25 & 0.35 \\
\hline 21 & 4.17 & 0.42 & 4.00 & 0.35 & 3.58 & 0.36 & 3.75 & 0.52 \\
\hline 22 & 4.00 & 0.46 & 3.33 & 0.53 & 5.00 & 0.37 & 4.08 & 0.40 \\
\hline 23 & 3.17 & 0.42 & 3.00 & 0.49 & 3.83 & 0.34 & 3.58 & 0.47 \\
\hline 24 & 1.75 & 0.22 & 2.17 & 0.44 & 2.42 & 0.40 & 2.33 & 0.40 \\
\hline 25 & 3.25 & 0.43 & 3.33 & 0.45 & 4.83 & 0.51 & 4.75 & 0.54 \\
\hline$M$ & 4.01 & 0.38 & 4.05 & 0.38 & 4.16 & 0.37 & 4.02 & 0.39 \\
\hline
\end{tabular}

Note-Correlation of means (trained/untrained): major second ascending, $r=.87(p<.001)$; major second descending, $r=.89(p<.001)$. 
Table A2

Descriptive Statistics for Ratings of Continuation Tones Following the Implicative Interval of a Minor Third

\begin{tabular}{|c|c|c|c|c|c|c|c|c|}
\hline \multirow[b]{3}{*}{$\mathrm{C}$} & \multicolumn{4}{|c|}{ Ascending } & \multicolumn{4}{|c|}{ Descending } \\
\hline & \multicolumn{2}{|c|}{ Trained } & \multicolumn{2}{|c|}{ Untrained } & \multicolumn{2}{|c|}{ Trained } & \multicolumn{2}{|c|}{ Untrained } \\
\hline & $M$ & $S E$ & $M$ & $S E$ & $M$ & $S E$ & $M$ & $S E$ \\
\hline 1 & 4.42 & 0.48 & 3.75 & 0.33 & 3.17 & 0.51 & 2.42 & 0.50 \\
\hline 2 & 2.33 & 0.40 & 2.25 & 0.30 & 2.25 & 0.49 & 2.08 & 0.45 \\
\hline 3 & 3.25 & 0.49 & 3.83 & 0.53 & 2.00 & 0.41 & 2.33 & 0.31 \\
\hline 4 & 2.75 & 0.25 & 3.25 & 0.30 & 4.50 & 0.44 & 3.92 & 0.36 \\
\hline 5 & 3.83 & 0.34 & 3.67 & 0.43 & 2.67 & 0.48 & 2.50 & 0.23 \\
\hline 6 & 5.92 & 0.23 & 5.00 & 0.46 & 3.75 & 0.41 & 4.00 & 0.35 \\
\hline 7 & 2.92 & 0.34 & 2.67 & 0.43 & 3.00 & 0.48 & 2.83 & 0.32 \\
\hline 8 & 4.75 & 0.45 & 5.25 & 0.22 & 4.17 & 0.46 & 4.67 & 0.45 \\
\hline 9 & 4.08 & 0.45 & 3.83 & 0.42 & 5.75 & 0.43 & 5.75 & 0.35 \\
\hline 10 & 4.83 & 0.34 & 5.17 & 0.44 & 4.67 & 0.47 & 3.83 & 0.49 \\
\hline 11 & 5.92 & 0.23 & 5.25 & 0.33 & 5.67 & 0.31 & 6.42 & 0.19 \\
\hline 12 & 4.17 & 0.60 & 4.33 & 0.51 & 4.17 & 0.37 & 3.67 & 0.41 \\
\hline 13 & 5.33 & 0.22 & 5.33 & 0.40 & 4.83 & 0.42 & 5.17 & 0.37 \\
\hline 14 & 3.50 & 0.47 & 4.25 & 0.46 & 5.67 & 0.28 & 4.83 & 0.34 \\
\hline 15 & 6.00 & 0.21 & 5.67 & 0.22 & 4.83 & 0.30 & 4.50 & 0.31 \\
\hline 16 & 3.92 & 0.42 & 3.83 & 0.39 & 5.25 & 0.35 & 6.25 & 0.30 \\
\hline 17 & 5.33 & 0.26 & 5.50 & 0.19 & 4.17 & 0.47 & 4.42 & 0.47 \\
\hline 18 & 6.58 & 0.19 & 5.75 & 0.33 & 5.67 & 0.26 & 5.67 & 0.19 \\
\hline 19 & 2.58 & 0.43 & 3.50 & 0.57 & 3.83 & 0.49 & 3.75 & 0.48 \\
\hline 20 & 6.08 & 0.29 & 5.83 & 0.21 & 4.67 & 0.41 & 5.33 & 0.40 \\
\hline 21 & 2.67 & 0.36 & 3.25 & 0.43 & 6.08 & 0.26 & 4.92 & 0.42 \\
\hline 22 & 5.58 & 0.40 & 5.58 & 0.47 & 3.17 & 0.39 & 3.42 & 0.47 \\
\hline 23 & 3.50 & 0.34 & 3.17 & 0.61 & 3.50 & 0.36 & 4.33 & 0.38 \\
\hline 24 & 1.75 & 0.28 & 2.58 & 0.53 & 2.08 & 0.31 & 2.58 & 0.45 \\
\hline 25 & 4.08 & 0.34 & 4.33 & 0.61 & 3.83 & 0.32 & 3.92 & 0.43 \\
\hline$M$ & 4.24 & 0.35 & 4.27 & 0.40 & 4.13 & 0.40 & 4.14 & 0.38 \\
\hline
\end{tabular}

Note - Correlation of means (trained/untrained): minor third ascending, $r=.93(p<.001)$; minor third descending, $r=$ $.89(p<.001)$

Table A3

Descriptive Statistics for Ratings of Continuation Tones Following the Implicative Interval of a Major Sixth

\begin{tabular}{|c|c|c|c|c|c|c|c|c|}
\hline \multirow[b]{3}{*}{$\mathrm{C}$} & \multicolumn{4}{|c|}{ Ascending } & \multicolumn{4}{|c|}{ Descending } \\
\hline & \multicolumn{2}{|c|}{ Trained } & \multicolumn{2}{|c|}{ Untrained } & \multicolumn{2}{|c|}{ Trained } & \multicolumn{2}{|c|}{ Untrained } \\
\hline & $M$ & $S E$ & $M$ & $S E$ & $M$ & $S E$ & $M$ & $S E$ \\
\hline 1 & 3.25 & 0.57 & 3.42 & 0.56 & 3.50 & 0.36 & 2.83 & 0.41 \\
\hline 2 & 4.08 & 0.40 & 4.08 & 0.48 & 1.50 & 0.26 & 2.33 & 0.31 \\
\hline 3 & 3.33 & 0.51 & 3.50 & 0.45 & 2.92 & 0.45 & 2.83 & 0.30 \\
\hline 4 & 5.00 & 0.30 & 4.00 & 0.48 & 2.33 & 0.41 & 1.92 & 0.23 \\
\hline 5 & 4.08 & 0.45 & 3.33 & 0.28 & 2.58 & 0.19 & 2.33 & 0.31 \\
\hline 6 & 4.58 & 0.42 & 4.08 & 0.38 & 5.25 & 0.46 & 4.33 & 0.45 \\
\hline 7 & 3.17 & 0.27 & 3.75 & 0.45 & 2.42 & 0.47 & 3.00 & 0.44 \\
\hline 8 & 3.75 & 0.28 & 4.25 & 0.30 & 3.83 & 0.34 & 3.50 & 0.36 \\
\hline 9 & 5.75 & 0.45 & 5.25 & 0.43 & 3.17 & 0.41 & 3.42 & 0.40 \\
\hline 10 & 3.42 & 0.40 & 3.33 & 0.38 & 5.00 & 0.37 & 4.50 & 0.38 \\
\hline 11 & 6.17 & 0.24 & 6.00 & 0.35 & 5.58 & 0.34 & 5.42 & 0.38 \\
\hline 12 & 4.33 & 0.38 & 4.42 & 0.47 & 4.83 & 0.39 & 4.67 & 0.47 \\
\hline 13 & 5.17 & 0.41 & 5.50 & 0.42 & 5.67 & 0.33 & 5.33 & 0.36 \\
\hline 14 & 5.83 & 0.21 & 5.33 & 0.40 & 4.67 & 0.31 & 3.92 & 0.45 \\
\hline 15 & 4.58 & 0.38 & 4.58 & 0.23 & 5.00 & 0.33 & 5.08 & 0.36 \\
\hline 16 & 5.33 & 0.40 & 5.75 & 0.30 & 5.00 & 0.48 & 4.83 & 0.32 \\
\hline 17 & 4.08 & 0.48 & 3.50 & 0.45 & 4.33 & 0.45 & 5.33 & 0.36 \\
\hline 18 & 4.17 & 0.41 & 5.25 & 0.30 & 6.58 & 0.23 & 5.42 & 0.34 \\
\hline 19 & 2.75 & 0.28 & 3.83 & 0.51 & 3.33 & 0.43 & 3.33 & 0.41 \\
\hline 20 & 4.00 & 0.35 & 4.75 & 0.41 & 6.25 & 0.18 & 6.25 & 0.18 \\
\hline 21 & 5.25 & 0.41 & 5.00 & 0.39 & 4.83 & 0.49 & 4.50 & 0.36 \\
\hline 22 & 2.50 & 0.38 & 3.58 & 0.57 & 5.67 & 0.28 & 5.08 & 0.51 \\
\hline 23 & 4.08 & 0.42 & 3.50 & 0.47 & 5.08 & 0.42 & 4.58 & 0.43 \\
\hline 24 & 2.00 & 0.37 & 2.17 & 0.52 & 3.00 & 0.49 & 3.25 & 0.33 \\
\hline 25 & 4.00 & 0.37 & 4.25 & 0.55 & 5.25 & 0.41 & 4.83 & 0.40 \\
\hline$M$ & 4.19 & 0.38 & 4.26 & 0.42 & 4.30 & 0.37 & 4.11 & 0.37 \\
\hline
\end{tabular}

Note-Correlation of means (trained/untrained): major sixth ascending, $r=.84(p<.001)$; major sixth descending, $r=$ $.93(p<.001)$. 
Table A4

Descriptive Statistics for Ratings of Continuation Tones Following the Implicative Interval of a Minor Seventh

\begin{tabular}{|c|c|c|c|c|c|c|c|c|}
\hline \multirow[b]{3}{*}{$\mathrm{C}$} & \multicolumn{4}{|c|}{ Ascending } & \multicolumn{4}{|c|}{ Descending } \\
\hline & \multicolumn{2}{|c|}{ Trained } & \multicolumn{2}{|c|}{ Untrained } & \multicolumn{2}{|c|}{ Trained } & \multicolumn{2}{|c|}{ Untrained } \\
\hline & $M$ & $S E$ & $M$ & $S E$ & $M$ & $S E$ & $M$ & $S E$ \\
\hline 1 & 4.67 & 0.38 & 5.00 & 0.37 & 3.75 & 0.41 & 3.00 & 0.46 \\
\hline 2 & 3.08 & 0.53 & 2.83 & 0.49 & 2.58 & 0.31 & 2.08 & 0.31 \\
\hline 3 & 4.08 & 0.45 & 4.25 & 0.48 & 2.08 & 0.29 & 2.83 & 0.42 \\
\hline 4 & 4.42 & 0.47 & 3.67 & 0.43 & 2.50 & 0.42 & 3.08 & 0.43 \\
\hline 5 & 4.42 & 0.34 & 4.33 & 0.45 & 2.33 & 0.28 & 2.25 & 0.28 \\
\hline 6 & 4.75 & 0.45 & 4.92 & 0.38 & 4.42 & 0.43 & 4.67 & 0.38 \\
\hline 7 & 3.42 & 0.53 & 3.42 & 0.57 & 2.17 & 0.34 & 2.58 & 0.26 \\
\hline 8 & 4.83 & 0.51 & 4.58 & 0.40 & 3.67 & 0.33 & 4.00 & 0.35 \\
\hline 9 & 3.42 & 0.43 & 3.42 & 0.38 & 3.92 & 0.48 & 4.17 & 0.32 \\
\hline 10 & 5.33 & 0.26 & 5.67 & 0.31 & 4.33 & 0.43 & 4.00 & 0.41 \\
\hline 11 & 5.17 & 0.41 & 5.33 & 0.40 & 4.58 & 0.36 & 5.50 & 0.31 \\
\hline 12 & 5.17 & 0.46 & 4.83 & 0.46 & 4.67 & 0.41 & 4.25 & 0.43 \\
\hline 13 & 4.75 & 0.37 & 5.08 & 0.53 & 4.83 & 0.41 & 5.25 & 0.33 \\
\hline 14 & 3.58 & 0.38 & 4.17 & 0.47 & 5.00 & 0.28 & 5.17 & 0.24 \\
\hline 15 & 5.42 & 0.38 & 5.25 & 0.35 & 4.42 & 0.31 & 4.92 & 0.31 \\
\hline 16 & 4.25 & 0.45 & 4.50 & 0.36 & 4.58 & 0.40 & 5.42 & 0.31 \\
\hline 17 & 3.92 & 0.47 & 4.75 & 0.33 & 3.92 & 0.29 & 4.67 & 0.43 \\
\hline 18 & 4.58 & 0.50 & 5.25 & 0.39 & 5.42 & 0.48 & 5.83 & 0.27 \\
\hline 19 & 3.92 & 0.40 & 3.58 & 0.42 & 3.75 & 0.39 & 3.42 & 0.43 \\
\hline 20 & 4.75 & 0.46 & 5.58 & 0.45 & 4.50 & 0.36 & 5.25 & 0.37 \\
\hline 21 & 3.33 & 0.43 & 3.83 & 0.42 & 5.58 & 0.38 & 5.67 & 0.58 \\
\hline 22 & 5.00 & 0.37 & 4.58 & 0.38 & 5.25 & 0.30 & 5.33 & 0.48 \\
\hline $2 \overline{3}$ & 2.50 & 0.45 & 3.17 & 0.47 & 4.33 & 0.33 & 4.83 & 0.55 \\
\hline 24 & 2.33 & 0.28 & 3.42 & 0.48 & 3.25 & 0.45 & 2.67 & 0.33 \\
\hline 25 & 4.33 & 0.45 & 4.33 & 0.64 & 4.92 & 0.36 & 5.08 & 0.50 \\
\hline$M$ & 4.22 & 0.42 & 4.39 & 0.43 & 4.03 & 0.37 & 4.24 & 0.38 \\
\hline
\end{tabular}

Note-Correlation of means (trained/untrained): minor seventh ascending, $r=.85(p<.001)$; minor seventh descending, $r=.92(p<.001)$.

(Manuscript received January 20, 1994;

revision accepted for publication December 10, 1994.) 